\title{
Total Glucosides of Danggui Buxue Tang Attenuate BLM-Induced Pulmonary Fibrosis via Regulating Oxidative Stress by Inhibiting NOX4
}

\author{
Ping Zhao, ${ }^{1}$ Wen-Cheng Zhou, ${ }^{2,3}$ De-Lin Li, ${ }^{1}$ Xiao-Ting Mo, ${ }^{3}$ Liang Xu, ${ }^{1}$ Liu-Cheng Li, ${ }^{3}$ \\ Wen-Hui Cui, ${ }^{3}$ and Jian Gao ${ }^{2}$ \\ ${ }^{1}$ School of Pharmacy, Anhui University of Chinese Medicine, Hefei 230038, China \\ ${ }^{2}$ The First Affiliated Hospital of Anhui Medical University, Hefei 230022, China \\ ${ }^{3}$ School of Pharmacy, Anhui Medical University, Hefei 230032, China \\ Correspondence should be addressed to Jian Gao; gaojianayfy@163.com
}

Received 28 March 2015; Revised 22 June 2015; Accepted 14 July 2015

Academic Editor: Domenico D’Arca

Copyright ( 2015 Ping Zhao et al. This is an open access article distributed under the Creative Commons Attribution License, which permits unrestricted use, distribution, and reproduction in any medium, provided the original work is properly cited.

\begin{abstract}
Pulmonary fibrosis $(\mathrm{PF})$ is a serious chronic lung disease with unknown pathogenesis. Researches have confirmed that oxidative stress which is regulated by NADPH oxidase-4 (NOX4), a main source of reactive oxygen species (ROS), is an important molecular mechanism underlying PF. Previous studies showed that total glucosides of Danggui Buxue Tang (DBTG), an extract from a classical traditional Chinese herbal formula, Danggui Buxue Tang (DBT), attenuated bleomycin-induced PF in rats. However, the mechanisms of DBTG are still not clear. We hypothesize that DBTG attenuates PF through regulating the level of oxidative stress by inhibiting NOX4. And we found that fibrosis indexes hydroxyproline (HYP) and type I collagen (Col-I) were lower in DBTG groups compared with the model group. In addition, the expression of transforming growth factor- $\beta 1$ (TGF- $\beta 1$ ) and expression of alpha smooth muscle actin ( $\alpha$-SMA) were also much more decreased than the model group. For oxidative stress indicators, DBTG blunted the decrease of superoxide dismutase (SOD) activity, total antioxidant capacity (T-AOC), and the increase in malondialdehyde (MDA), 8-iso-prostaglandin in lung homogenates. Treatment with DBTG restrained the expression of NOX4 compared to the model group. Present study confirms that DBTG inhibits BLM-induced PF by modulating the level of oxidative stress via suppressing NOX4.
\end{abstract}

\section{Introduction}

Pulmonary fibrosis (PF) is a chronic lung disease and characterized by excessive accumulation of extracellular matrix (ECM) deposition to mesenchymal transition (EMT), which finally leads to the decline of lung function [1]. Because environmental pollution is more and more serious, the incidence of PF is on the rise in China recently [2]. Although the mechanisms of PF are not understood fully, transforming growth factor- $\beta 1$ (TGF- $\beta 1$ ) is recognized as a critical factor to induce fibrosis [3]. More and more researches have confirmed that oxidative stress is an important molecular mechanism underlying fibrosis in a variety of organs, especially for lung [4]. After being stimulated by dust, drugs, and other factors, oxidative stress usually occurred in lung and the balance between oxides and peroxides will be broken; then, the level of reactive oxygen species (ROS) will be increased because of oxidative stress. ROS induces PF by promoting apoptosis of alveolar epithelial cells (AECs), inflammatory cell infiltrating, accumulation of collagen, and ECM [5]. Oxidative stress is frequently defined as the imbalance of oxidant production and antioxidant defenses and leads to cellular dysfunction and tissue damage [6]. NOX4 belongs to the NADPH oxidase (NOX) family of enzymes, which catalyze the reduction of $\mathrm{O}_{2}$ to form ROS [7]. It is one of the most important sources of ROS in vivo. Researches have shown that NOX4 is closely associated with the development of PF comparing to other enzymes in NOX family [8].

Danggui Buxue Tang (DBT) composed of Radix Astragali (RA) and Radix Angelicae Sinensis (RAS) at a ratio of 5:1 
is a classical traditional Chinese herbal formula [9]. DBT is traditionally used by women as a remedy for menopausal symptoms, in China [9]. Nowadays, recent pharmacological researches confirmed that DBT had the ability to improve haematopoietic function, stimulate cardiovascular circulation, prevent osteoporosis, increase antioxidation activity, and stimulate the immune system $[10,11]$. Total glucosides of Danggui Buxue Tang (DBTG) is an extract from DBT. Our previous studies have confirmed that DBTG attenuated bleomycin- (BLM-) induced PF in rats via inhibition of ECM $[12,13]$. Since the mechanisms of DBTG are still unclear, we hypothesize that DBTG attenuates PF through regulating the level of oxidative stress by inhibiting NOX4. The results of the present study may explore other potential mechanisms of DBTG and provide the basis for the clinical treatment of PF.

\section{Materials and Methods}

2.1. Animals. Sprague-Dawley rats weighing $180-220 \mathrm{~g}(n=$ 210) were obtained from the Laboratory Animal Department of Anhui Medical University, Hefei, China. Rats were housed at a constant room temperature $\left(23 \pm 2^{\circ} \mathrm{C}\right)$, humidity $(60 \pm$ $10 \%)$, and light cycle (12:12 h light-dark), with free access to tap water as well as standard chow. All rats' experimental procedures were approved by Anhui Medical University Animal Care Committee and followed the protocol outlined in The Guide for the Care and Use of Laboratory Animals published by the USA National Institute of Health (NIH Pub. No. 85-23, Revised 1996).

2.2. Preparation of DBTG and Content Determination. Two different medicinal herbs that consist of DBT were purchased from the pharmacy of The First Affiliated Hospital of Anhui Medical University, China, and all of them were identified by Professor Wang De-qun (Anhui College of Traditional Chinese Medicine, Hefei, China) as Astragali Radix [root of Astragalus membranaceus the (Fisch.) Bge. var. mongholicus (Bge.) Hsiao (Huangqi, 14021203)] and Angelicae Sinensis Radix [root of Angelicae Sinensis (Oliv.) Diels (Danggui, 13041502)]. The method of extraction DBTG was reported in our previous study $[9,10]$. In brief, DBTG would be extracted by the following method. Astragalus membranaceus and Radix Angelicae sinensis were soaked in water for an hour and reflux was then heated three times ( $2 \mathrm{~h}$ per time). The extract would be filtered, enriched, and placed in $4^{\circ} \mathrm{C}$ for one night. The second day, the extract was centrifuged in $3000 \mathrm{r}$ and impurities were removed. The supernatant was used to extract DBTG through macroporous silica gel D101. First water was used to remove the polysaccharide substance by slowly flushing silica gel column. Other impurities were discarded by $40 \%$ alcohol after water was flushed. The solution after $80 \%$ alcohol flushed was saved. DBTG would be got after drying the $80 \%$ alcohol solution. The content and fingerprint of DBTG were detected as the methods that previously described in Chinses $[14,15]$. The average content of DBTG was $70.7 \%$.

2.3. Experiment Design. Animals were randomly divided into the following seven groups ( $n=30$ per group): group 1 [control + no treatment], group 2 [sham-operated (sham) group received only NS + no treatment], group 3 [a dose of $5 \mathrm{mg} \cdot \mathrm{kg}^{-1}$ bleomycin (BLM) induced PF model + no treatment], group 4/5/6 [a dose of $5 \mathrm{mg} \cdot \mathrm{kg}^{-1} \mathrm{BLM}$ induced PF model + a single oral dose of $4 / 8 / 16 \mathrm{mg} \cdot \mathrm{kg}^{-1}$ DBTG treatment], and group 7 [a dose of $5 \mathrm{mg} \cdot \mathrm{kg}^{-1} \mathrm{BLM}$ induced PF model + a single oral dose of $5 \mathrm{mg} \cdot \mathrm{kg}^{-1}$ prednisone treatment].

Bleomycin-A5 (Harbin Laiboten Pharmaceutical Co. LTD., Harbin, China) was dissolved by sterile normal saline (NS) with a volume of $1.6 \mathrm{~mL}$ before PF modeling. Animals in 3,4,5 and 6 group were treated with BLM at a dose of $5 \mathrm{mg} \cdot \mathrm{kg}^{-1}$ by intratracheal instillation. And animals in group 2 were just treated with sterile NS in the same mode of administration. DBTG and prednisone $(5 \mathrm{mg} \times 100$ tablets, no. 1210246, Xinhua Pharmaceutical CO., LTD, Shandong, China) were dissolved by $0.5 \%$ CMC-Na the day before administration. The first day after modeling, 0.5\% CMC$\mathrm{Na}\left(1.2 \mathrm{~mL} \cdot \mathrm{kg}^{-1}\right)$ was given to rats in groups 1,2 , and 3 by intragastric administration (ig) once daily. Groups 3, 5, and 6 were treated with DBTG $\left(4 / 8 / 16 \mathrm{mg} \cdot \mathrm{kg}^{-1}\right.$, per day, ig) and rats received prednisone $\left(5 \mathrm{mg} \cdot \mathrm{kg}^{-1}\right.$, per day, ig). Body weight of rats was recorded daily and animals were not sacrificed if they showed weight loss above a certain threshold. Rats were sacrificed with anaesthetizing by an intraperitoneal injection of pentobarbital $(40 \mathrm{mg} / \mathrm{kg})$ on days 7,14 , and 28 after modeling and then samples were obtained.

2.4. Sample Collection and Handling. Lung tissues were collected on days 7, 14, and 28 after modeling. Lung tissue was flushed with saline until no blood in a dish, and then the lung was weighed. Lung coefficient (lung weight (mg)/body weight (g)) would be calculated at each time point. Half of the left lung was fixed in $4 \%$ phosphate-buffered paraformaldehyde for histopathologic and immunohistochemistry preparation, while the other was frozen after using for homogenate. Lung tissues were homogenized in at $4^{\circ} \mathrm{C}$ saline three times with a polytron homogenizer (10 s of homogenization at $10 \mathrm{~s}$ intervals). And supernatant was reserved after homogenate centrifuged at $3000 \mathrm{~g}$ for 10 minutes and stored at $-80^{\circ} \mathrm{C}$ for further analysis.

2.5. Histopathologic and Immunohistochemical. Lung samples were fixed in 10\% phosphate-buffered formalin for $24 \mathrm{~h}$. The median portion was cut and embedded in paraffin. The paraffin blocks were cut at $5 \mu \mathrm{m}$ using a microtome and deparaffinized tissue slices were subjected to Masson and Hematoxylin and Eosin (H\&E) for histological examination. Immunohistochemical was used to investigate the expression of $\alpha$-SMA, Col-I, and TGF- $\beta 1$. In brief, after being deparaffinized, paraffin sections were treated with $1.0 \%$ periodate for $1 \mathrm{~min}$ to block endogenous peroxidase activity. Then paraffin sections were treated with citrate buffer in a microwave oven for $10 \mathrm{~min}$ (antigen retrieval). Sections were washed by phosphate buffered saline (PBS) three times ( $5 \mathrm{~min}$ per time) once cooled to room temperature. After washing, sections were blocked with normal goats serum for $30 \mathrm{~min}$ 
at $37^{\circ} \mathrm{C}$, followed with primary antibodies against the $\alpha$ SMA antigen (1: 600, ab5694, Abcam, Cambridge, UK), Col-I antigen (1:700, ab34710, Abcam, Cambridge, UK), and TGF$\beta 1$ antigen (1:50, ab25121, Abcam, Cambridge, UK) overnight at $4^{\circ} \mathrm{C}$. Negative control group was made by the remaining slide. Next day, after washing the slides thrice with PBS (5 min per time), sections were incubated with secondary antibody for $10 \mathrm{~min}$ at $37^{\circ} \mathrm{C}$. PBS washed and sections were treated with streptavidin-biotin-peroxidase complex for 10 min. After washing, diaminobenzidine (DAB) was added as a visualizing agent. Hematoxylin was used for nuclear staining. The sections were examined under light microscope at a photodocumentation facility (Olympus, Tokyo). For each tissue section, the number of positively stained cells was calculated from ten different 400x magnified fields under light microscope.

2.6. Content of HYP, Col-I, SOD, MDA, GSH, 8-Iso-Prostaglandin, and T-AOC in Lung Homogenates. The content of HYP in lung homogenate was measured by HYP kits (A030-2, Nanjing Jiancheng Biochemical Institute, Nanjing, China) as our previous study [12-14]. Col-I and 8-isoprostaglandin were all tested by ELISA kits (Col-I: 41582, 8iso-prostaglandin: 41874, Yuanye Bioengineering, Shanghai, China). SOD and T-AOC were determined by measuring the absorbance at 550 and $520 \mathrm{~nm}$ by using detection kits (SOD: A001-1, T-AOC, A015, Nanjing Jiancheng Biochemical Institute, Nanjing, China). The MDA content was observed by the thiobarbituric acid reactive substances (TBARS) assay in accordance with the manufacturer's instructions (MDA: A003-1, Jiancheng Biochemical Institute, Nanjing, China) and the absorbance was measured at a wavelength of $532 \mathrm{~nm}$.

2.7. Western Blotting Analysis. Lung tissue samples were weighed (80-100 mg) on days 7, 14, and 28 and homogenized on ice with ice-cold lysis buffer (P0013C, Beyotime Institute of Biotechnology, China) including protease inhibitor, PMSF (Amresco 0754, Biosharp, USA) for $30 \mathrm{~min}$. After centrifugation $\left(12,000 \mathrm{r} / \mathrm{min}, 10 \mathrm{~min}\right.$ at $\left.4^{\circ} \mathrm{C}\right)$, the supernatant was collected, packed, and stored in $-80^{\circ} \mathrm{C}$. Just before using, the loading buffer was mixed into the supernatant at a ratio of $1: 4$ and cooked in boiling water for $10 \mathrm{~min}$. Proteins in the supernatant were separated by SDS-PAGE on a $12 \%$ gel and then transferred to PVDF membranes (no. 20130107054, Millipore, USA). The blotted membranes were blocked with 5\% nonfat dry milk (w/v) (No. 2013022311, Guangming, China). After 2 hours, blotted membranes were washed by $0.2 \%$ Tween-X100 (TBST) three times ( $10 \mathrm{~min}$ per time). Then blotted membranes were incubated at $4^{\circ} \mathrm{C}$ overnight with anti- $\alpha$-SMA (1:100, ab5694, Abcam, Cambridge, UK), antiNOX4 (1:5000, ab133303, Abcam, Cambridge, UK), and anti$\beta$-actin (1:5000, ab32572, Abcam, Cambridge, UK) antibodies. Next day PVDF membranes were blocked with 5\% nonfat dry milk with Peroxidase Conjugated AffniPure Goat AntiRabbit lgG (1: 15000, ZB-2301, ZSGB-BIO, Beijing, China) for $1 \mathrm{~h}$. After being washed with TBST three times (10 min per time), the blots were visualized with ECL reagent (Thermo Scientific, Rockford, IL, USA).
2.8. Statistical Analysis. The data are expressed as mean \pm SEM. For all the statistical tests, multiple comparisons were performed by one way analysis of variance (ANOVA) with the Tukey-Kramer or Tamhane's tests by SPSS 17.0. In addition, Graphpad prism 5 software was used for plotting graphs. Values of $p<0.05$ and $p<0.01$ were considered to be statistically significant.

\section{Results}

3.1. Effect of DBTG on PF in Rats. In order to show the role of DBTG on PF, indexes of PF like H\&E staining, Masson staining, content of HYP, and Col-I were all tested. Results from H\&E staining (Figure 1(a)) and Masson staining (Figure 1(b)) indicated that DBTG could reduce the degree of PF compared with the model group. In addition, the content of HYP (Figure 1(c)) and Col-I (Figure $1(\mathrm{~d})$ ) of rats with DBTG treatment was lower than the model group significantly $(p<$ 0.05 or $p<0.01)$.

3.2. Effect of DBTG on Expression of TGF- $\beta 1$ and $\alpha$-SMA. TGF- $\beta 1$ and its downstream regulatory protein $\alpha$-SMA are important signs of PF. They were detected by immunohistochemistry or western-blot analysis. In Figures 2(a) and 2(b), the expression of TGF- $\beta 1$ and $\alpha$-SMA could be inhibited in groups that were treated with DBTG by immunohistochemistry testing. At the same time, the expression of $\alpha$ SMA was tested by western-blot analysisat each time point (Figures 2(c), 2(d), and 2(e)). After modeling, $\alpha$-SMA was obviously increased compared to control and sham group $(p<0.05$ or $p<0.01)$. Treated with DBTG, the expression of $\alpha$-SMA was much lower than the model group on days 7 , 14 , and $28(p<0.05$ or $p<0.01)$.

3.3. Effect of DBTG on the Level of Oxidative Stress in Rats with PF. The levels of MDA, 8-iso-prostaglandin, SOD, and T-AOC (Figure 3 ) were detected to observe the degree of oxidative stress in lung tissue after modeling. Results from Figure 3 showed that the balance of oxidative stress was broken in PF rats compared with the control and sham group ( $p<0.05)$. Apart from this, the rats treated with DBTG demonstrated the reduced oxidative stress indicators like MDA (Figure 3(a)), 8-iso-prostaglandin (Figure 3(b)), and the upregulated antioxidant indicators such as T-SOD (Figure 3(c)) and T-AOC (Figure 3(d)) in contrast with the model group $(p<0.05$ or $p<0.01)$.

3.4. Effect of DBTG on Expression of NOX4. NOX4 plays a critical role in oxidative stress. To investigate the effect of DBTG on NOX4, western-blot analysis was performed (Figure 4). The expression of NOX4 was significantly decreased especially on days 7 and 14 after BLM modeling compared with control and sham group. ( $p<0.05$ or $p<$ $0.01)$. These results indicated that DBTG could inhibit NOX4. 

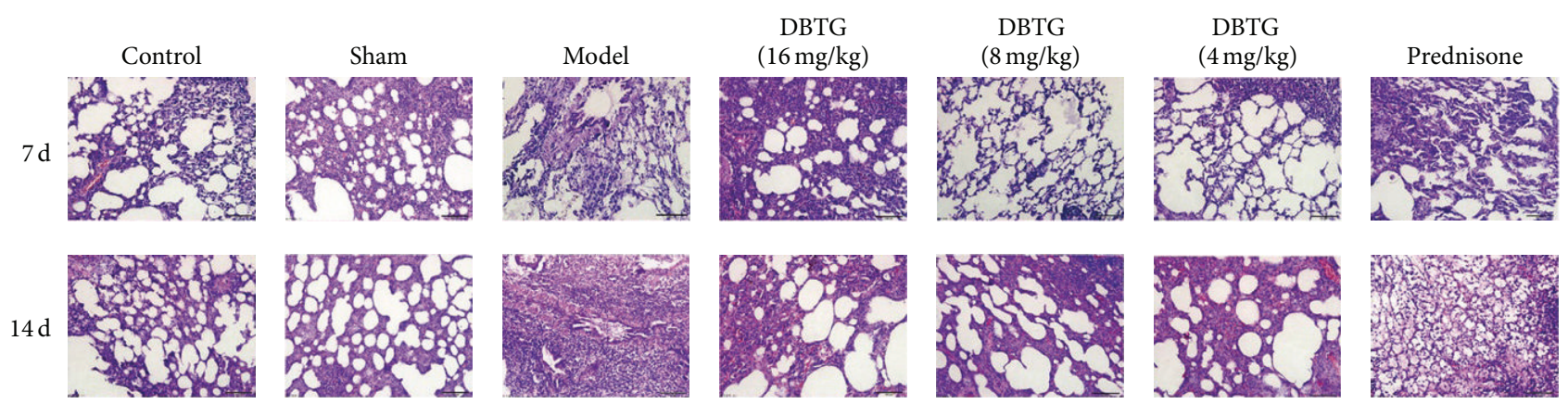

$28 \mathrm{~d}$
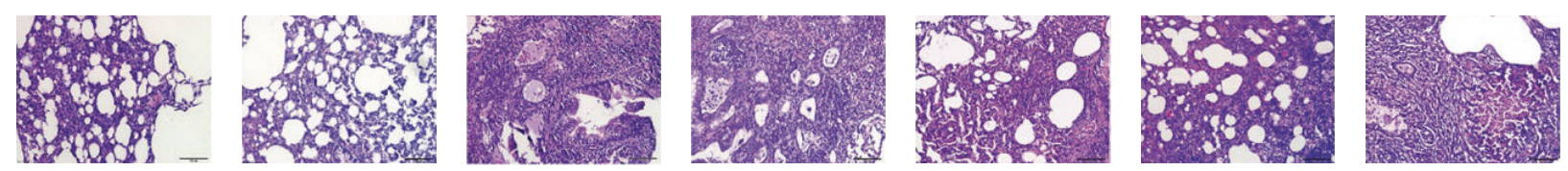

(a)
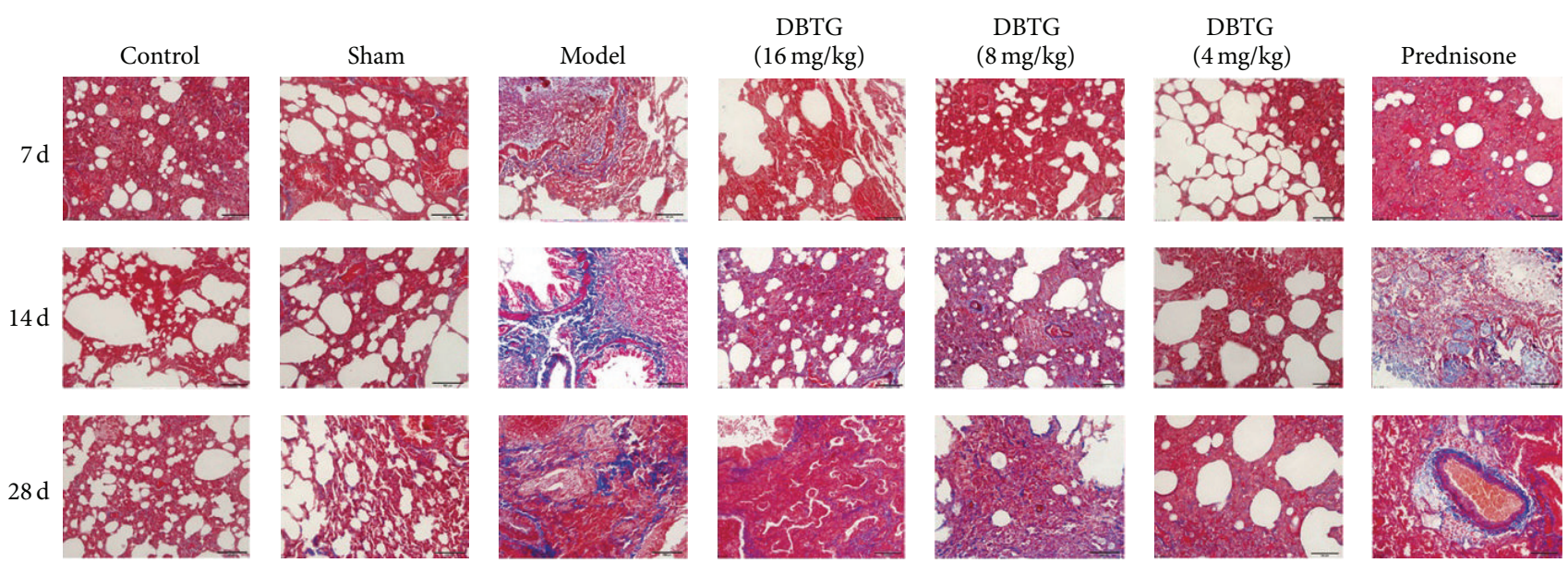

(b)

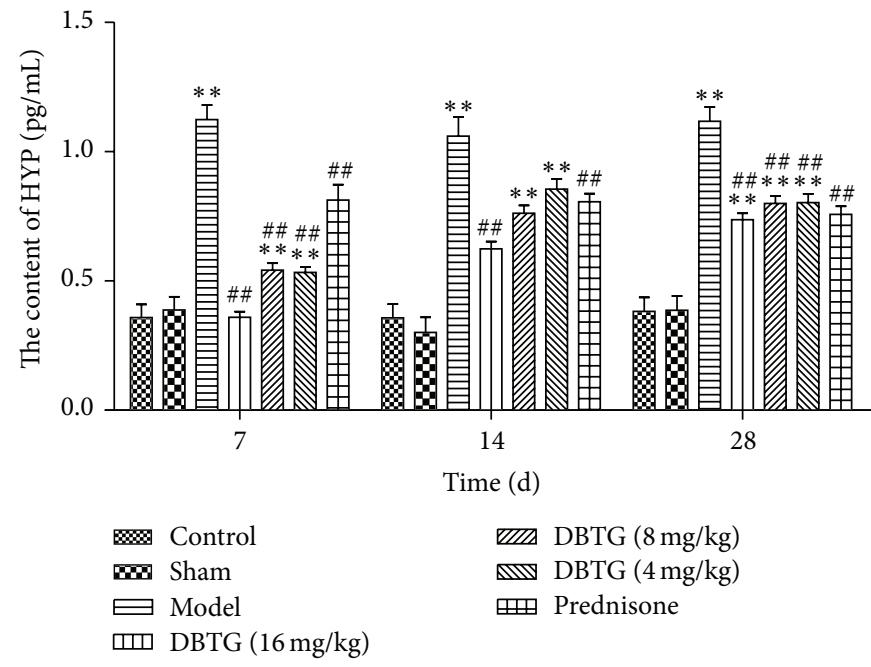

(c)

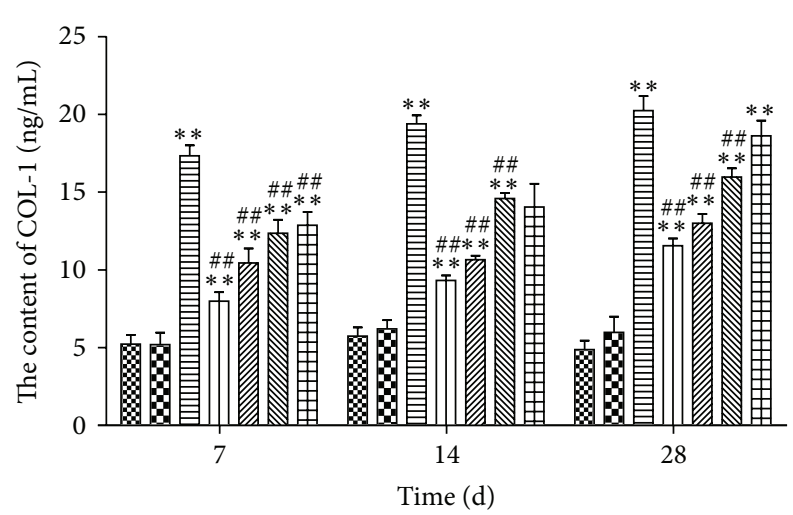

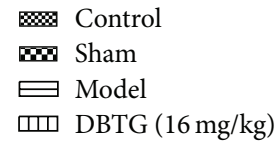

(d)

FIGURE 1: Effect of DBTG on lung fibrosis in BLM-induced rats PF. (a) H\&E staining of rat lung tissues (400x); (b) Masson staining of rat lung tissues (400x); (c) content of HYP in lung tissues; (d) content of COL-I in lung homogenates. DBTG could reduce the degree of PF. Data were expressed as mean \pm SEM, $n \geq 5,{ }^{*} p<0.05$ versus control group, ${ }^{* *} p<0.01$ versus control group; ${ }^{*} p<0.05$ versus model group, ${ }^{* \#} p<0.01$ versus model group. 

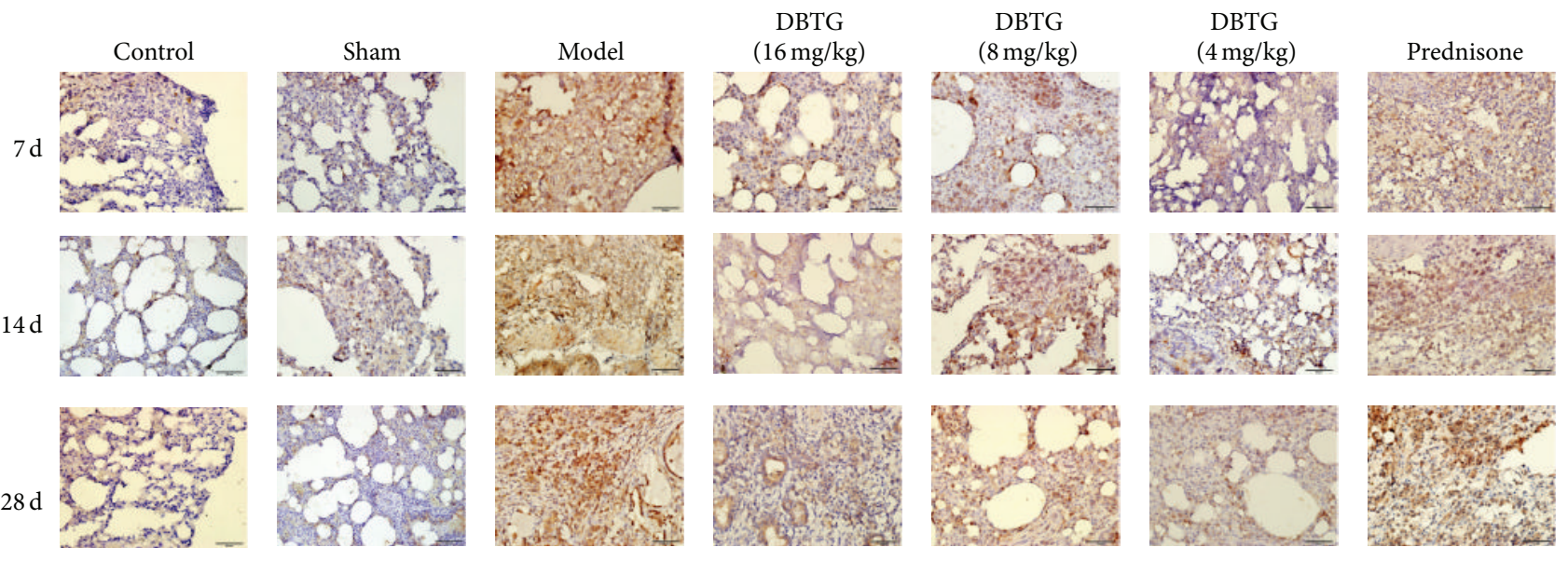

(a)
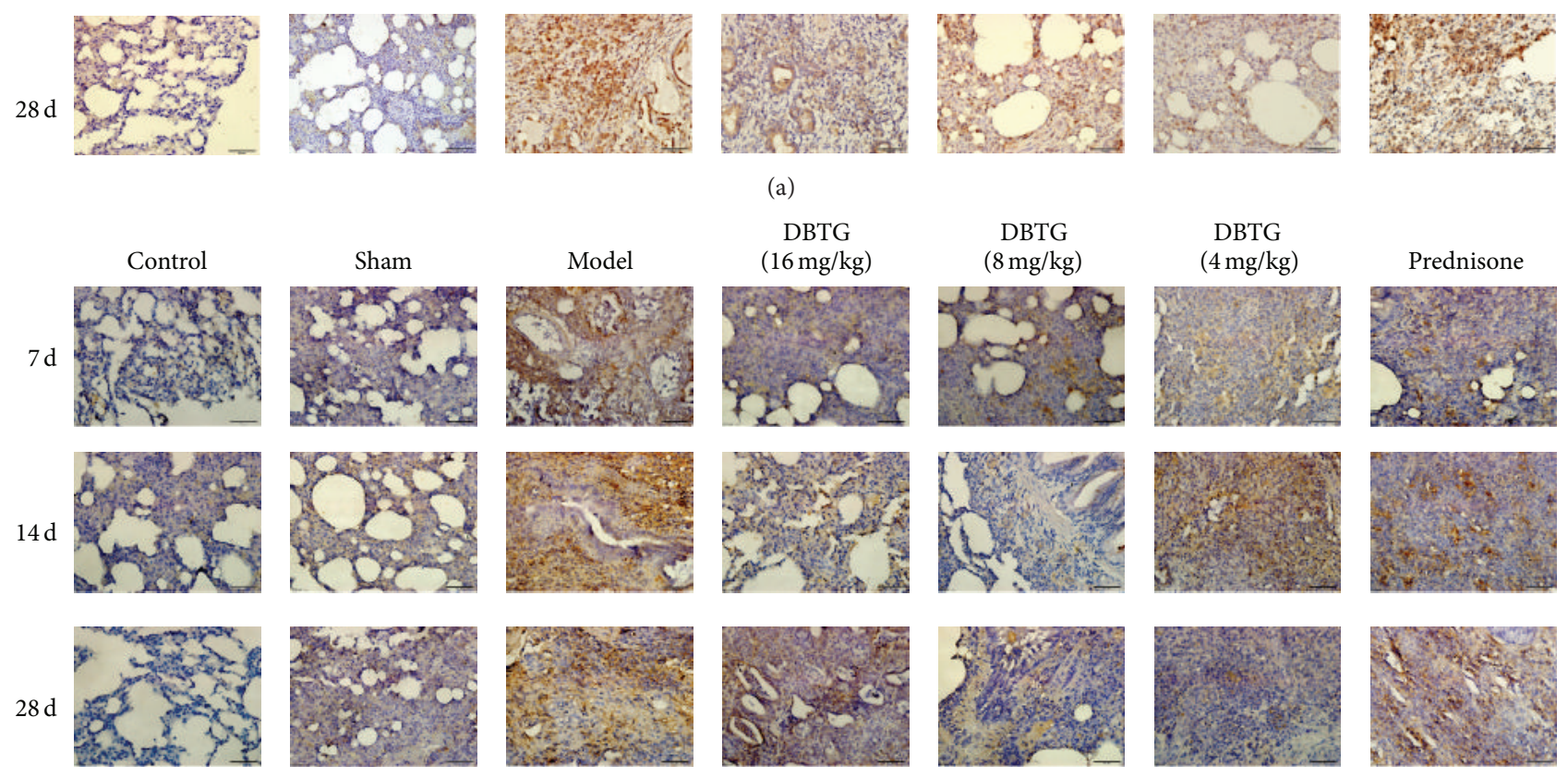

(b)
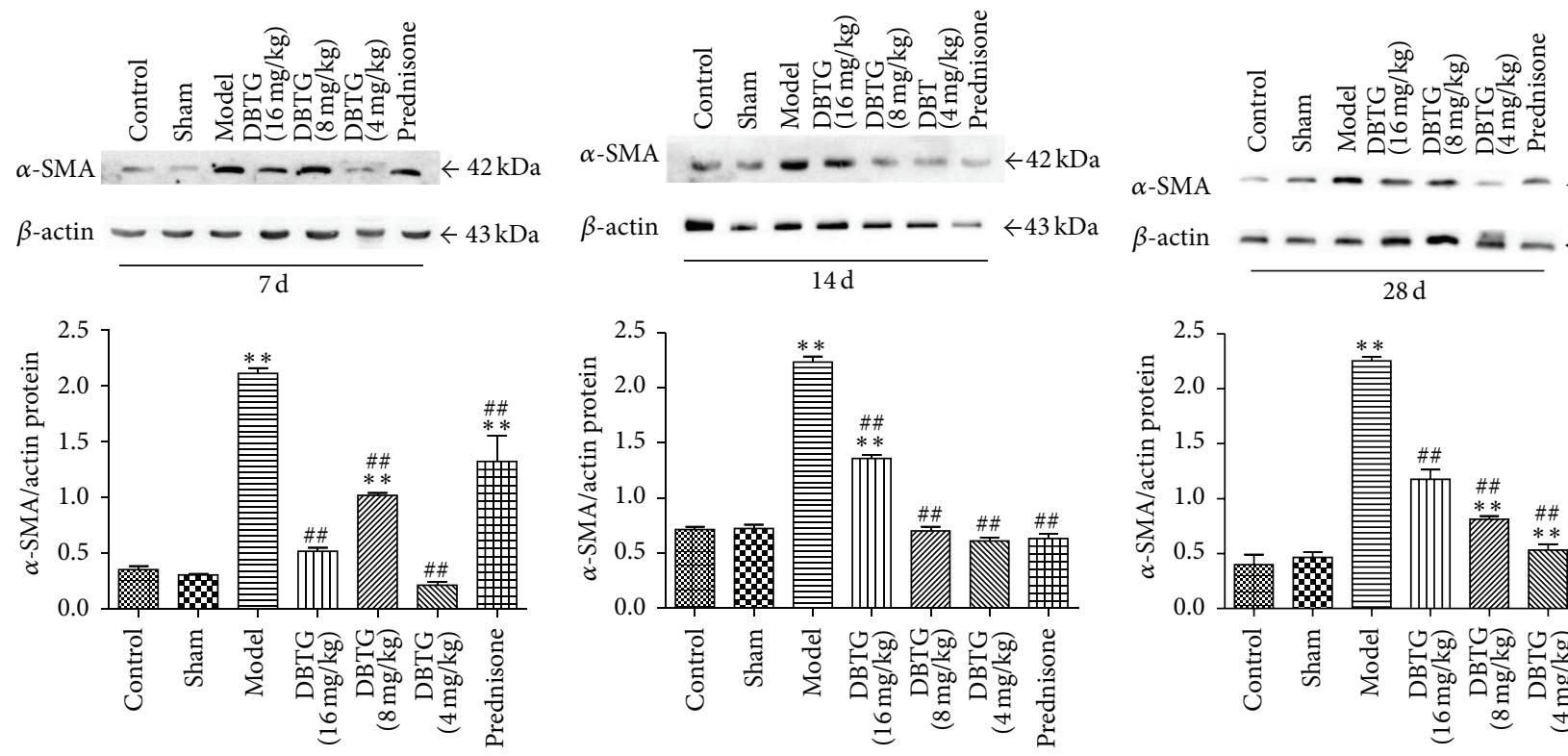

(d)

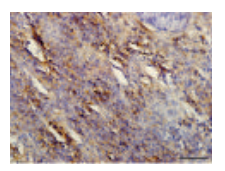

(c)

FIGURE 2: Effect of DBTG on expression of TGF- $\beta 1$ and $\alpha$-SMA in BLM-induced rats PF. (a) Immunohistochemistry of TGF- $\beta 1$ (100x); (b) immunohistochemistry of $\alpha$-SMA (100x); (c) representative western-blots of $\alpha$-SMA, $\beta$-actin at 7 days $(n=3)$; (d) representative westernblots of $\alpha$-SMA, $\beta$-actin at 14 days $(n=3)$; (e) representative western-blots of $\alpha$-SMA, $\beta$-actin at 28 days $(n=3)$. The expression of TGF- $\beta 1$ and $\alpha$-SMA were downregulated by DBTG comparing to model group. Data were expressed as mean \pm SEM, $n \geq 5,{ }^{*} p<0.05$ versus control group, ${ }^{* *} p<0.01$ versus control group; ${ }^{\#} p<0.05$ versus model group, ${ }^{\#} p<0.01$ versus model group. 

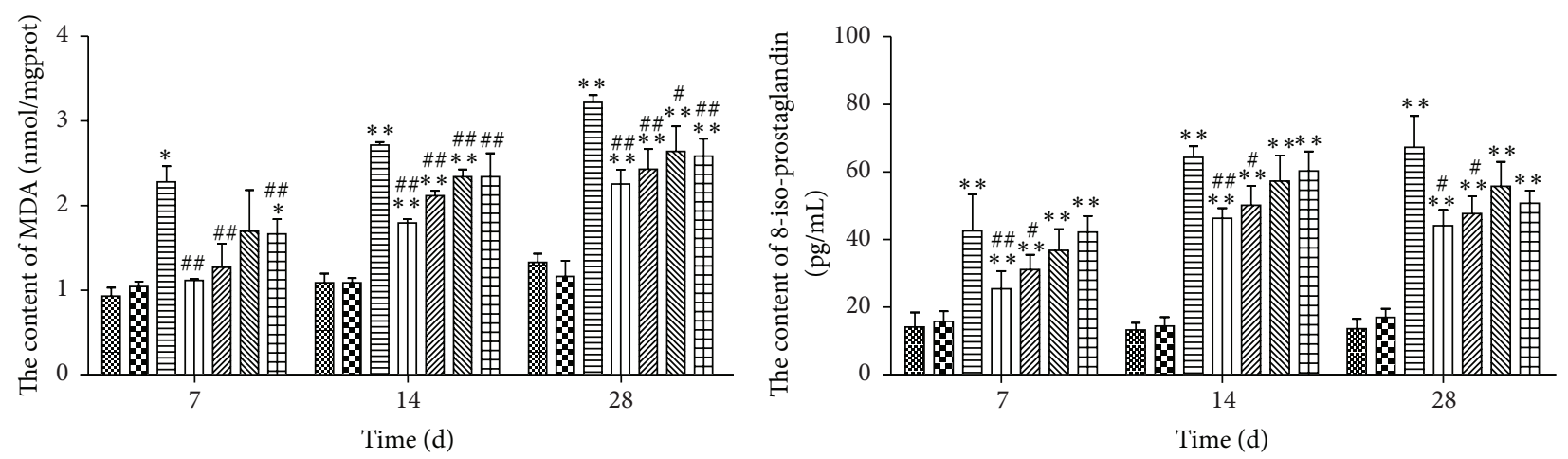

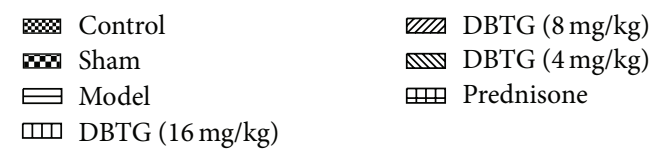

(a)
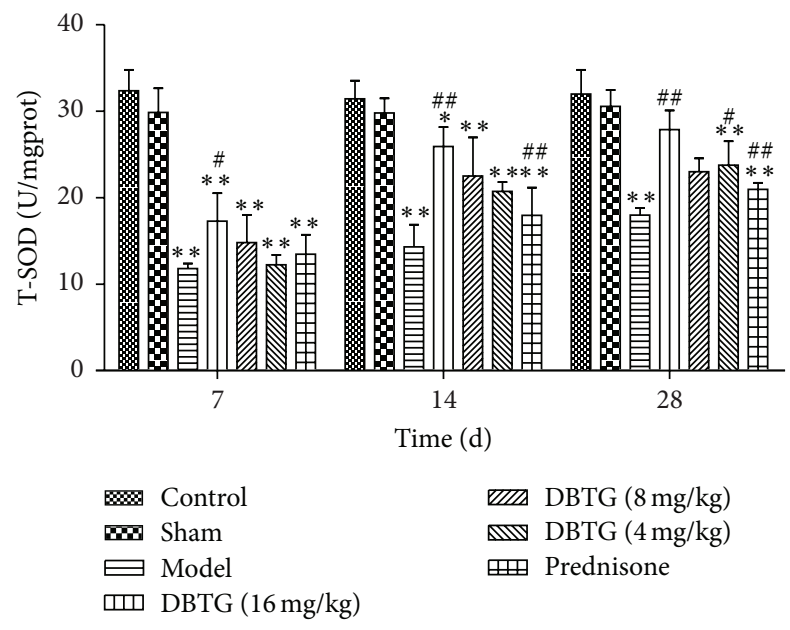

(c)

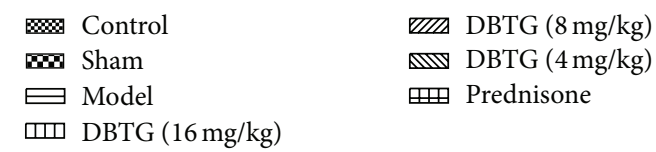

(b)
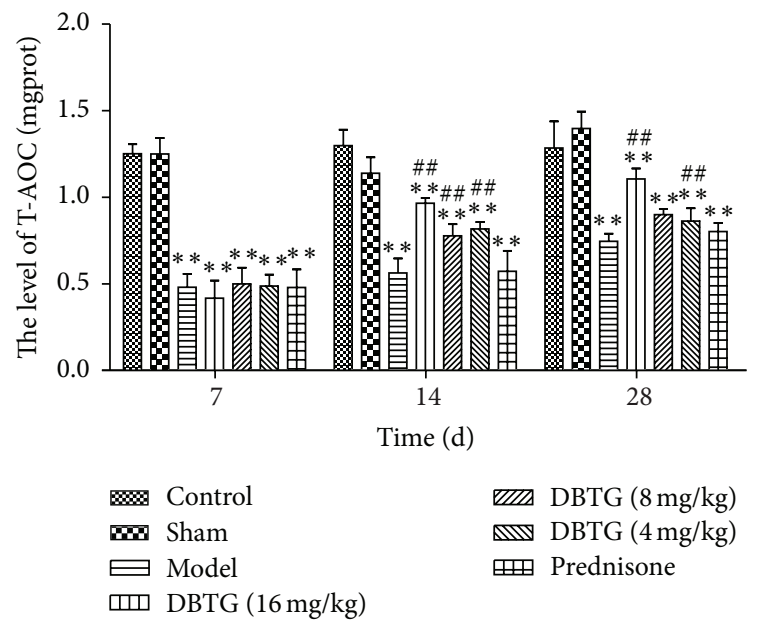

(d)

FIGURE 3: Effect of DBTG on the level of oxidative stress in BLM-induced rats PF. (a) Content of MDA in lung homogenates; (b) content of 8-iso-prostagland in lung homogenates; (c) content of T-SOD in lung homogenates; (d) level of T-AOC in lung homogenates. Oxidative stress indicators like MDA and 8-iso-prostaglandin were reduced and antioxidant indicators such as T-SOD and T-AOC were increased by treatment of DBTG. Data were expressed as mean \pm SEM, $n \geq 5,{ }^{*} p<0.05$ versus control group, ${ }^{* *} p<0.01$ versus control group; ${ }^{*} p<0.05$ versus model group, ${ }^{\# \#} p<0.01$ versus model group.

\section{Discussion}

$\mathrm{PF}$ is a serious progressive chronic lung disease that is incurable in current treatment and usually results in death [16]. Until now, the pathogenesis of PF remains unknown. So far, there is no effective drug for the treatment of PF. Since the current treatments of PF like anti-inflammatory or anti-immune are ineffective, so drugs with curative and low toxicity are urgently needed.

Before researching the effective antifibrotic medicine, pathogenesis of PF should be understood clearly. At present, the mechanism of PF is unclear and there are mainly several different theories [17-19]. In spite of these theories, TGF- $\beta 1$ is currently recognized as one of the most important profibrotic growth factors in the development of PF [1]. Besides that, studies have also confirmed that inflammation factors such as Th1/Th2, IL7, and NF- $\kappa \mathrm{B}$ contributed to the process of
PF [20-22]. With the deepening research of oxidative stress, recent evidences have showed that oxidative stress plays an important role in PF $[23,24]$. When body is subjected to external stimuli, oxidative stress will be induced. Because when oxidative stress happened, reactive oxygen species (ROS) and antioxidants were imbalanced and excessive ROS could damage the normal, physiological function of proteins, lipids, nucleic acids, and other macromolecules in many different cells directly or indirectly. NOX4 belongs to the NADPH oxidase (NOX) family of enzymes that catalyze the reduction of $\mathrm{O}_{2}$ to form ROS and it might promote the progress of PF by increasing differentiation of myofibroblast [5].

DBTG is one of the active components isolated from DBT. Our previous studies suggested that DBTG attenuated BLMinduced PF in rats via inhibition of ECM $[12,13]$. Since the relationship between DBTG and oxidative stress is unclear in 

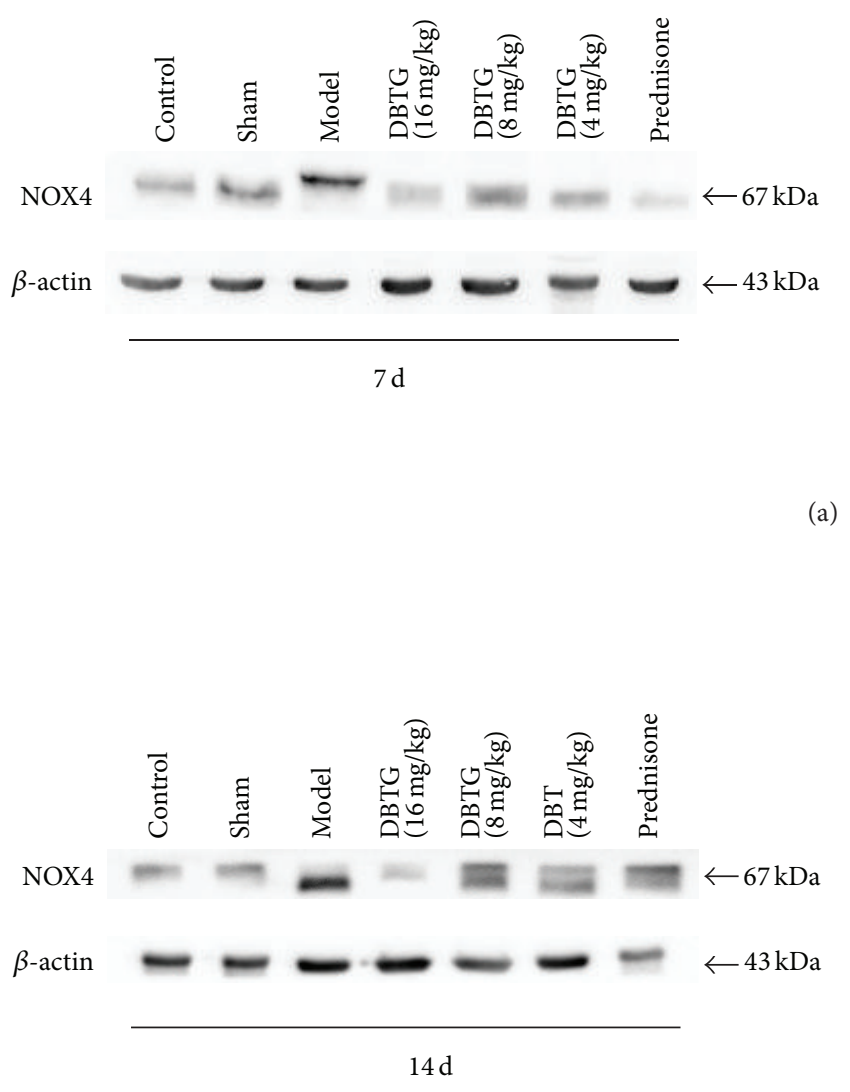

(a)
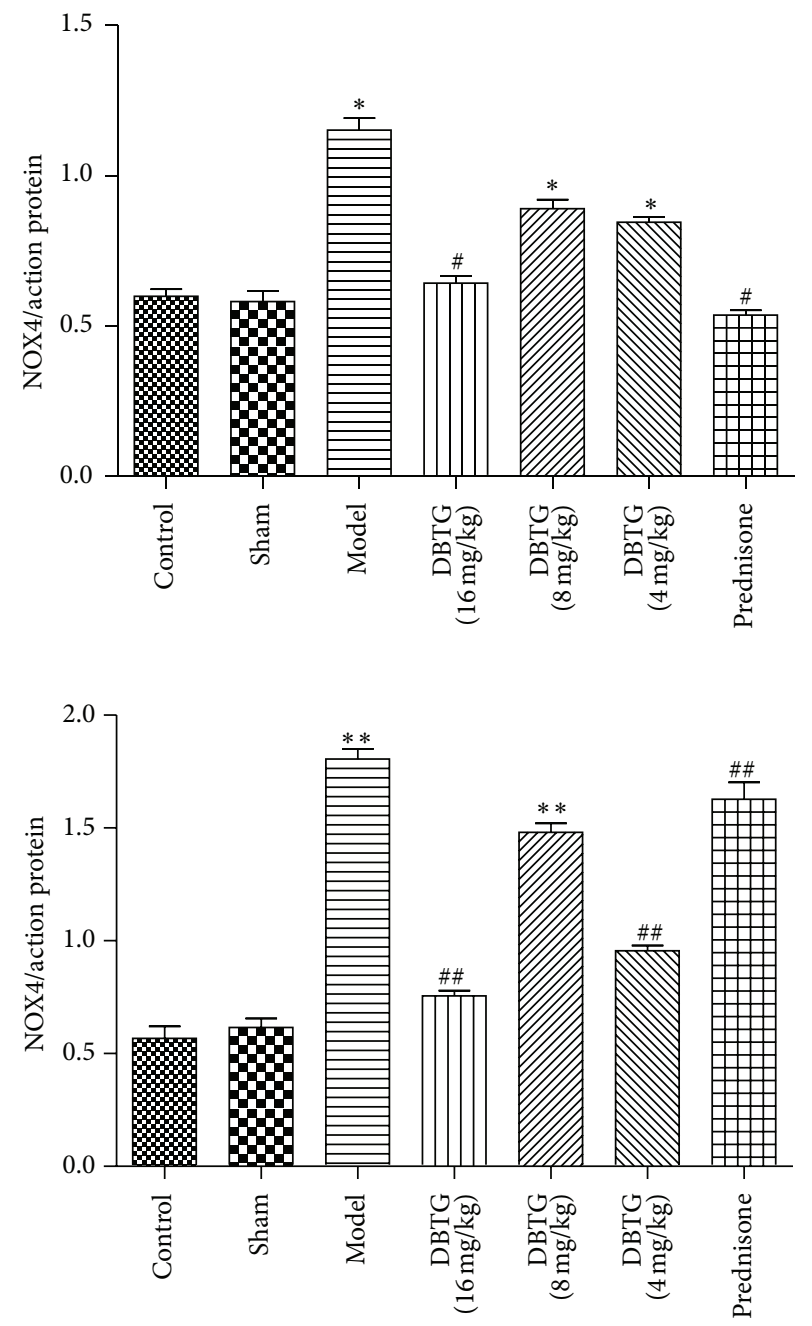

(b)

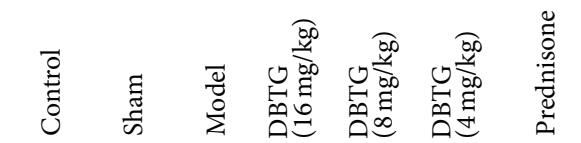

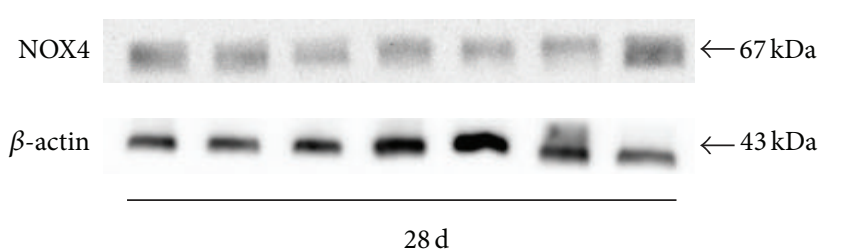

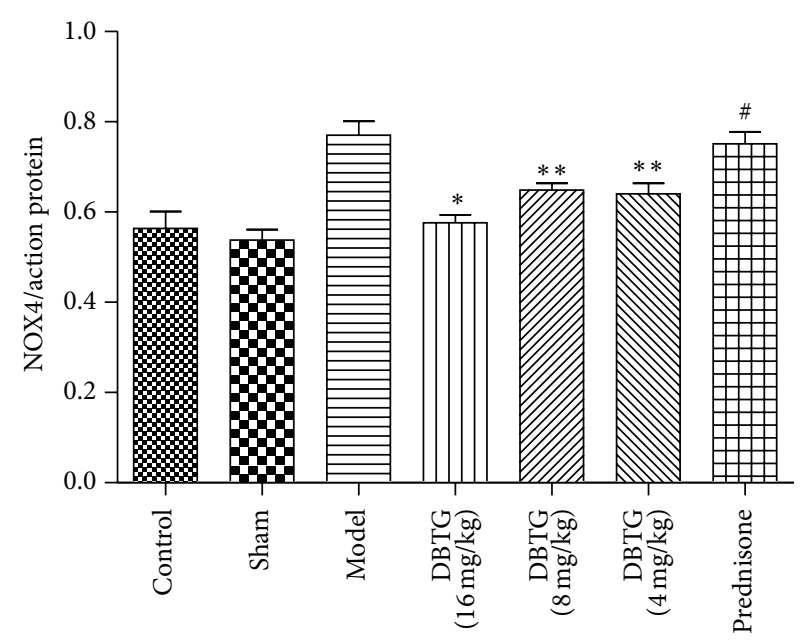

(c)

FIGURE 4: Effect of DBTG on expression of NOX4 in BLM-induced rats PF. (a) Representative western-blots of NOX4 and $\beta$-actin at 7 days $(n=3)$; (b) representative western-blots of NOX 4 and $\beta$-actin at 14 days $(n=3)$; (c) representative western-blots of NOX4 and $\beta$-actin at 28 days $(n=3)$. The expression of NOX4 was inhibited in DBTG treatment groups contrast with model group. Data were expressed as mean \pm SEM, ${ }^{*} p<0.05$ versus control group, ${ }^{* *} p<0.01$ versus control group; ${ }^{\#} p<0.05$ versus model group, ${ }^{\# \#} p<0.01$ versus model group. 


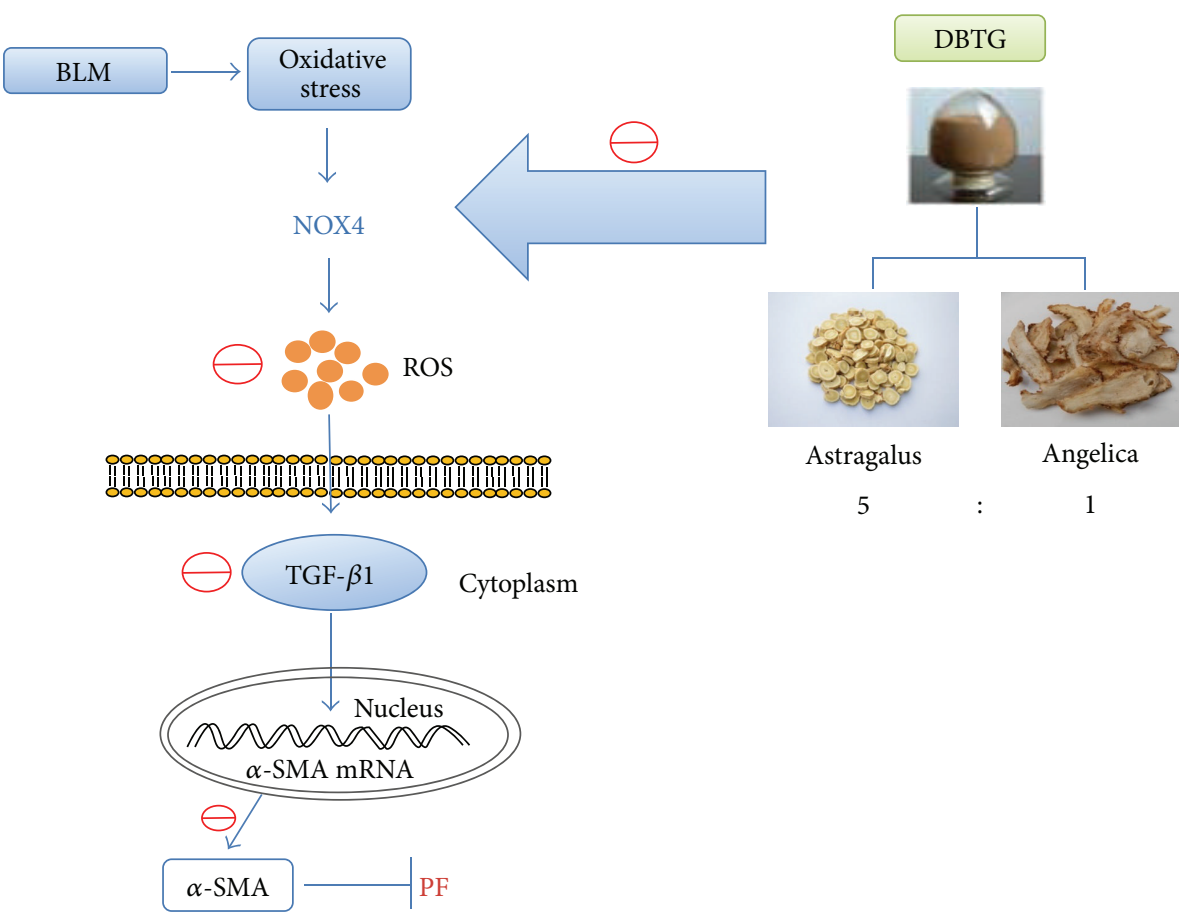

FIGURE 5: DBTG attenuates BLM-induced rats PF via regulating oxidative stress by inhibiting NOX4. In this study, oxidative stress happened in BLM-induced PF. And then, a lot of ROS was synthesized by NOX4 to promote the expression of TGF- $\beta 1$ and its downstream protein $\alpha$-SMA that increased the degree of PF. DBTG could inhibit NOX4 to reduce ROS and control the level of oxidative stress. As the level of oxidative stress was regulated, the expression of TGF- $\beta 1$ and expression of $\alpha$-SMA were both decreased and, at last, the development of PF could be inhibited.

PF, in this study, we explored whether DBTG affects oxidative stress via suppressing NOX4 to inhibit PF. From results of H\&E (Figure 1(a)) and Masson staining (Figure 1(b)), the model of PF in rats was successful. Compared with model group, DBTG significantly reduced the content of HYP (Figure 1(c)) and Col-I (Figure 1(d)), especially for the dose of $16 \mathrm{mg} \cdot \mathrm{kg}^{-1}$. The results proved that DBTG had the ability to mitigate the degree of PF. TGF- $\beta 1$ is a critical factor in PF and $\alpha$-SMA is a downstream protein of TGF- $\beta 1$. They were tested by immunohistochemical and western-blot (Figure 2). In Figure 2, TGF- $\beta 1$ was inhibited with treatment of DBTG and the expression of $\alpha$-SMA was downregulated.

To analyze the level of oxidative stress, MDA, 8-isoprostaglandin, SOD, and T-AOC were detected by assay kits (Figure 3). It was confirmed that DBTG could increase the level of antioxidant indexes (SOD, T-AOC) and decrease the level of peroxide indexes (MDA, 8-iso-prostaglandin). DBTG might control the development of PF by regulating the level of oxidative stress. And DBTG with the dose of $16 \mathrm{mg} \cdot \mathrm{kg}^{-1}$ was one of the strongest control efforts on regulating the level of oxidative stress. Because NOX4 plays an important role in oxidative stress, it was detected by western-blot at each time point (Figure 4). NOX4 was increased after modeling. This may be related to the stress response in rats themselves. And DBTG might decrease the expression of NOX4. As NOX4 plays an important role in oxidative stress, DBTG might protect against $\mathrm{PF}$ via controlling the level of oxidative stress by inhibiting NOX4 (Figure 5).
Based on these results we found that the lowest dose should be more effective than the other two. We analyzed that the reasons might be as follows. First, DBTG is an extract from Chinese medicine that has nonsingle active ingredients like Astragaloside II and Astragaloside IV. Different active ingredients and doses usually play different effects. Second, mechanism of traditional Chinese medicine is multitarget and this will influence the efficiency of treatment. Third, the lower dose may have less toxicity that is helpful to treatment. So the lowest dose of DBTG might be more effective than others.

This study demonstrated that DBTG attenuated BLMinduced pulmonary fibrosis via regulating oxidative stress by inhibiting NOX4. However, the mechanism of DBTG inhibiting NOX4 is still unknown and needs more studies in further future.

\section{Conclusions}

All the above findings suggested that DBTG could attenuate the degree of BLM-induced PF in rats via inhibiting NOX4 to control the level of oxidative stress. NOX4 may be a potential target for inhibiting PF and DBTG may be a promising candidate for the prevention and treatment of $\mathrm{PF}$.

\section{Abbreviations \\ AECs: Alveolar epithelial cells BLM: Bleomycin}


Col-I: Type I collagen

DBT: Danggui Buxue Tang

DBTG: Total glucosides of Danggui Buxue Tang

PF: Pulmonary fibrosis

ECL: Electrochemiluminescence

ECM: Extracellular matrix

EMT: Epithelial to mesenchymal transition

ROS: Reactive oxygen species

H\&E: Hematoxylin and eosin

HYP: Hydroxyproline

MDA: Malondialdehyde

NOX4: NADPH oxidase- 4

ROS: Reactive oxygen species

Sham: Sham-operated rats

SOD: Superoxide dismutase

T-AOC: Total antioxidant capacity

TGF-1: Transforming growth factor- $\beta 1$

$\alpha$-SMA: Alpha smooth muscle actin.

\section{Disclosure}

Ping Zhao, Wen-Cheng Zhou, and De-Lin Li are co-first authors.

\section{Conflict of Interests}

The authors declare that there is no conflict of interests regarding the publication of this paper.

\section{Authors' Contribution}

Ping Zhao, Wen-Cheng Zhou, and De-Lin Li contributed equally to this paper.

\section{Acknowledgments}

This work was financially supported by the National Natural Science Foundation of China (nos. 81473267, 81274172, and 30801535) and Traditional Chinese Medicine Research Project of the Health Department of Anhui Province (no. 2012zy53).

\section{References}

[1] M. Gharaee-Kermani, B. Hu, S. H. Phan, and M. R. Gyetko, "Recent advances in molecular targets and treatment of Idiopathic Pulmonary Fibrosis: focus on TGF $\beta$ signaling and the myofibroblast," Current Medicinal Chemistry, vol. 16, no. 11, pp. 1400-1417, 2009.

[2] L. Nalysnyk, J. Cid-Ruzafa, P. Rotella, and D. Esser, "Incidence and prevalence of idiopathic pulmonary fibrosis: review of the literature," European Respiratory Review, vol. 21, no. 126, pp. 355-361, 2012.

[3] H. I. Adamali and T. M. Maher, "Current and novel drug therapies for idiopathic pulmonary fibrosis," Drug Design, Development and Therapy, vol. 6, pp. 261-272, 2012.

[4] B. M. Hybertson, B. Gao, S. K. Bose, and J. M. McCord, "Oxidative stress in health and disease: the therapeutic potential of Nrf2 activation," Molecular Aspects of Medicine, vol. 32, no. 46, pp. 234-246, 2011.

[5] P. Cheresh, S.-J. Kim, S. Tulasiram, and D. W. Kamp, "Oxidative stress and pulmonary fibrosis," Biochimica et Biophysica ActaMolecular Basis of Disease, vol. 1832, no. 7, pp. 1028-1040, 2013.

[6] S. Piera-Velazquez and S. A. Jimenez, "Role of cellular senescence and NOX4-mediated oxidative stress in systemic sclerosis pathogenesis," Current Rheumatology Reports, vol. 17, article 473, 11 pages, 2014.

[7] L. Hecker, R. Vittal, T. Jones et al., "NADPH oxidase-4 mediates myofibroblast activation and fibrogenic responses to lung injury," Nature Medicine, vol. 15, no. 9, pp. 1077-1081, 2009.

[8] L. Hecker, N. J. Logsdon, D. Kurundkar et al., "Reversal of persistent fibrosis in aging by targeting Nox4-Nrf2 redox imbalance," Science Translational Medicine, vol. 6, no. 231, Article ID 231ra47, 2014.

[9] P. Wang, Y. Liang, N. Zhou et al., "Screening and analysis of the multiple absorbed bioactive components and metabolites of Dangguibuxue decoction by the metabolic fingerprinting technique and liquid chromatography/diode-array detection mass spectrometry," Rapid Communications in Mass Spectrometry, vol. 21, no. 2, pp. 99-106, 2007.

[10] L. Fang, C. Cheng, T. Hwang et al., "Danggui buxue tang inhibits 2,4-dinitrochlorobenzene: induced atopic dermatitis in mice," Evidence-Based Complementary and Alternative Medicine, vol. 2015, Article ID 672891, 10 pages, 2015.

[11] A. G. Gong, N. Li, K. Lau et al., "Calycosin orchestrates the functions of Danggui Buxue Tang, a Chinese herbal decoction composing of Astragali Radix and Angelica Sinensis Radix: an evaluation by using calycosin-knock out herbal extract," Journal of Ethnopharmacology, vol. 168, pp. 150-157, 2015.

[12] J. Gao, Y. Huang, P. Li et al., "Antifibrosis effects of total glucosides of Danggui-Buxue-Tang in a rat model of bleomycininduced pulmonary fibrosis," Journal of Ethnopharmacology, vol. 136, no. 1, pp. 21-26, 2011.

[13] J. Gao, L.-J. Feng, Y. Huang et al., “Total glucosides of Danggui Buxue Tang attenuates bleomycin-induced pulmonary fibrosis via inhibition of extracellular matrix remodelling," Journal of Pharmacy and Pharmacology, vol. 64, no. 6, pp. 811-820, 2012.

[14] J. Gao, Q. Xia, Z. G. Huang, D. J. Xu, and J. Li, "Simultaneous assay of astragaloside IV and astragaloside II in total glucosides of Danggui Buxue Decoction by HPLC-ELSD," Chinese Traditional Patent Medicine, vol. 34, pp. 268-272, 2012 (Chinese).

[15] Z. G. Huang, J. Gao, P. Li et al., "Fingerprint of total glucosides of Danggui Buxue Tang by HPLC," Traditional Patent Medicine, vol. 32, pp. 1284-1286, 2010 (Chinese).

[16] Z. H. Hoo and M. K. B. Whyte, "Idiopathic pulmonary fibrosis," Thorax, vol. 67, no. 8, pp. 742-746, 2012.

[17] N. W. Todd, I. G. Luzina, and S. P. Atamas, "Molecular and cellular mechanisms of pulmonary fibrosis," Fibrogenesis and Tissue Repair, vol. 5, no. 1, article 11, 2012.

[18] L. T. Tang, T. Jiang, X. L. Han, and D. L. Chen, "Effect of tranilast on bleomycin induced pulmonary fibrosis in a rat model," African Journal of Pharmacy and Pharmacology, vol. 5, no. 10, pp. 1315-1320, 2011.

[19] T. H. Sisson, M. Mendez, K. Choi et al., "Targeted injury of type II alveolar epithelial cells induces pulmonary fibrosis," American Journal of Respiratory and Critical Care Medicine, vol. 181, no. 3, pp. 254-263, 2010.

[20] V. L. Kinnula and M. Myllärniemi, "Oxidant-antioxidant imbalance as a potential contributor to the progression of human 
pulmonary fibrosis," Antioxidants and Redox Signaling, vol. 10, no. 4, pp. 727-738, 2008.

[21] Y. Cui, J. Robertson, S. Maharaj et al., "Oxidative stress contributes to the induction and persistence of TGF-betal induced pulmonary fibrosis," International Journal of Biochemistry and Cell Biology, vol. 43, no. 8, pp. 1122-1133, 2011.

[22] E. R. Jarman, V. S. Khambata, C. Cope et al., "An Inhibitor of NADPH oxidase-4 attenuates established pulmonary fibrosis in a rodent disease model," American Journal of Respiratory Cell and Molecular Biology, vol. 50, no. 1, pp. 158-169, 2014.

[23] C. R. Kliment and T. D. Oury, "Oxidative stress, extracellular matrix targets, and idiopathic pulmonary fibrosis," Free Radical Biology and Medicine, vol. 49, no. 5, pp. 707-717, 2010.

[24] O. R. Temneanu, C. Zamfir, F. E. Zugun, E. Cojocaru, and L. Tocan, "Oxidants and antioxidants relevance in rats' pulmonary induced oxidative stress," Journal of Medicine and Life, vol. 4, no. 3, pp. 244-249, 2011. 


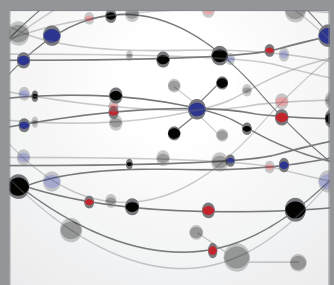

The Scientific World Journal
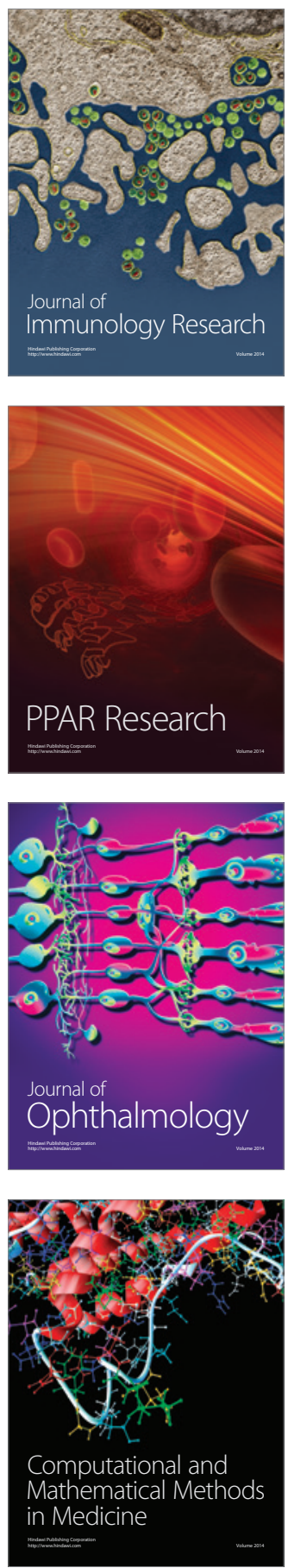

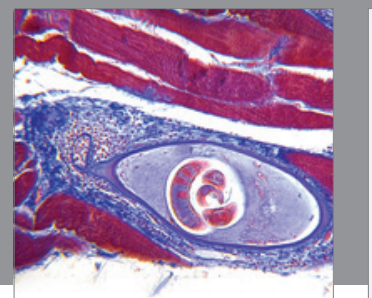

Gastroenterology

Research and Practice
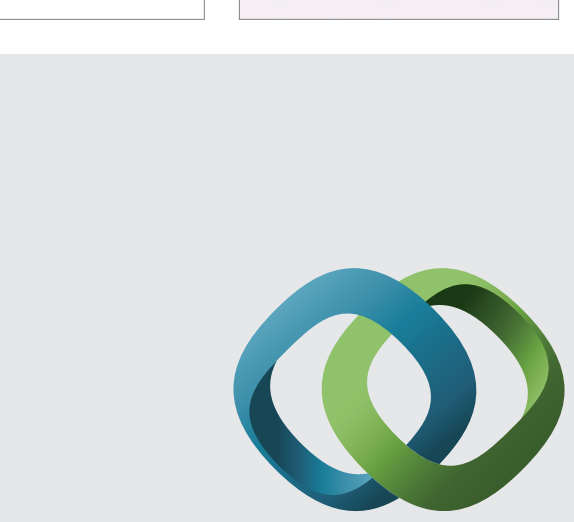

\section{Hindawi}

Submit your manuscripts at

http://www.hindawi.com
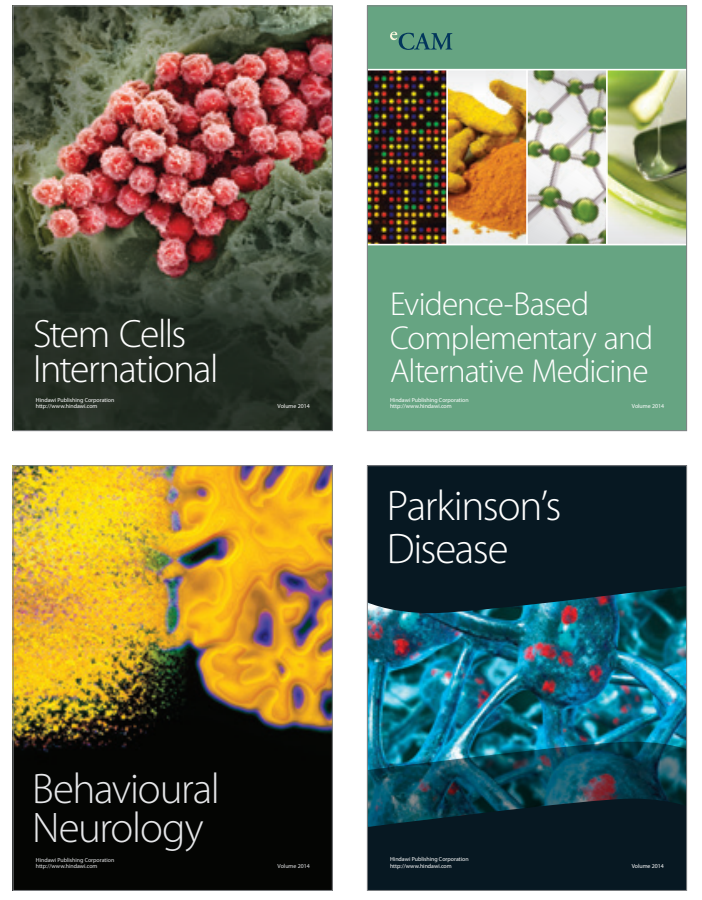
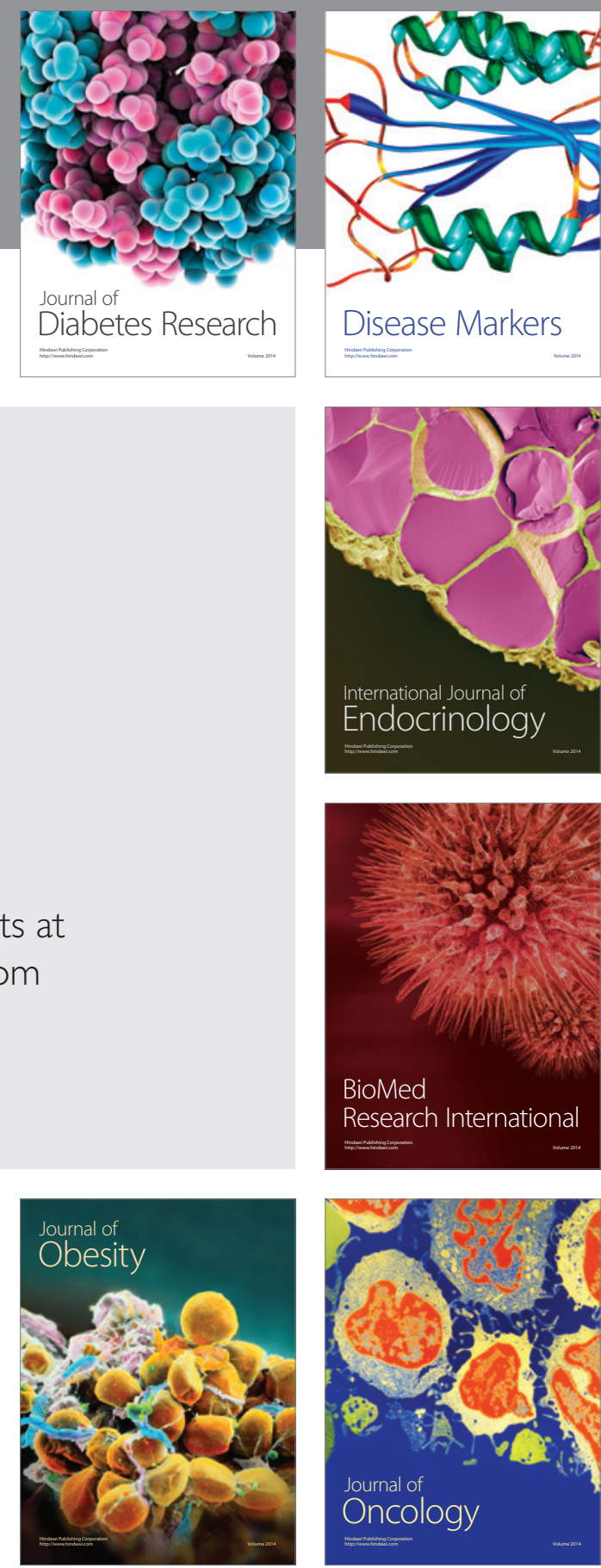

Disease Markers
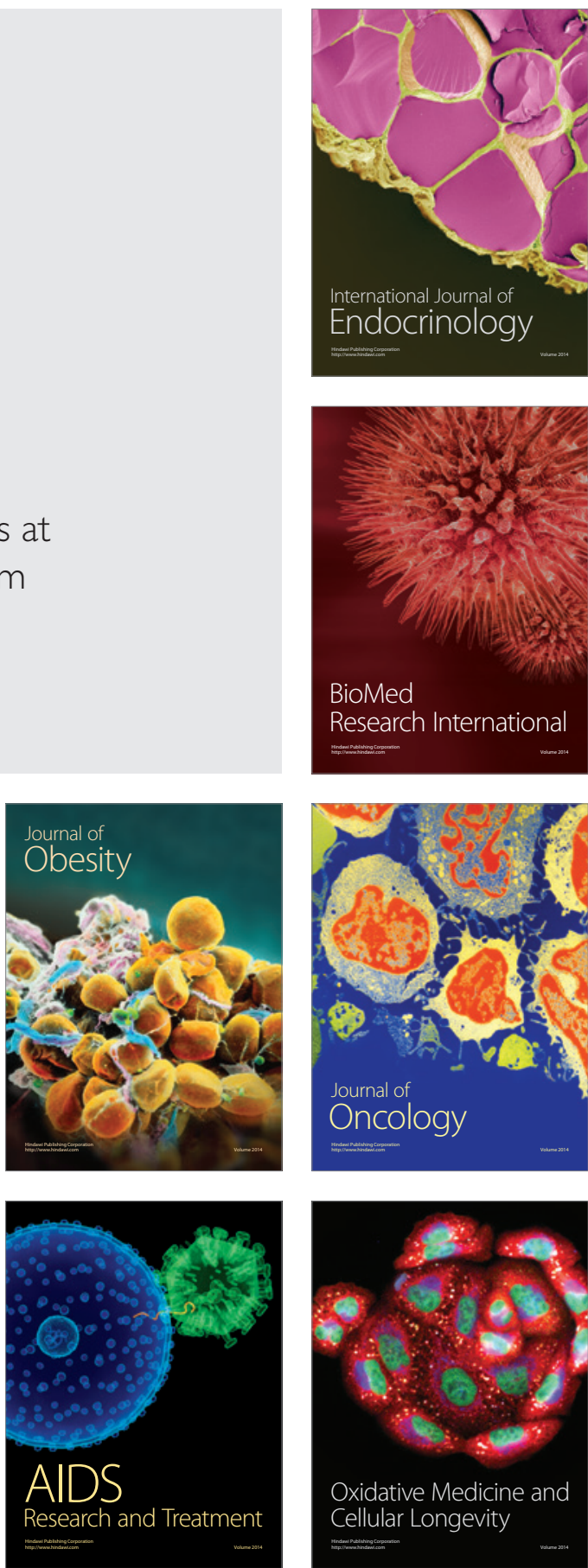